intestinal tract are described, and are well illustrated by case reports, photographs, and line drawings. The chapter on oesophagus, stomach and duodenum includes a description of the techniques of gastric fundal patch, pleural wrap, and diaphragmatic patch for the repair of postoperative oesophageal leaks, and the use of a Foley catheter, omental wrap of Roux-en-Y loop for a leaking duodenal stump following a Polya (Billroth II) gastrectomy. For lateral duodenal fistula, there is a good description with clear drawings of the techniques of serosal patching and jejunal pedicle grafting. The final two chapters include the management of biliary leakage, hepatic abscess, and the use of temporary enterostomy and intestinal intubation in early postoperative small bowel obstruction with sepsis. The management of small bowel cutaneous fistula is also described, together with the treatment of large bowel anastomosis leakage. The book concludes with a final reminder of the high mortality of IAS, of which a large proportion is due to errors in surgical technique and judgement. The authors correctly leave the reader in no doubt as to the Surgeon's responsibility in the prophylaxis of this condition. Professor Aeberhard and Dr. Casey are to be congratulated on this first class book which provides both the general surgeon and ITU physician with an excellent guide to the prevention, diagnosis and treatment of postoperative intraabdominal sepsis.

Rodney T. Croft (London)

Acute Respiratory Failure. W. M. Zapol, K. J. Falke (eds). New York Basle: Dekker 1985. Approx. 641 pp. ISBN 0-8247-7235-0

This is the twenty-fourth volume in the series 'Lung Biology in Health and Disease' which has Claude Lenfant as executive editor. The high standard of production seen in previous issues has been maintained, particularly the quality of illustrations depicting angiographic findings and electron micrographs. Inevitably of course, this is reflected in the price. The title is marginally misleading in that the entire volume is devoted to aspects of the adult respiratory distress syndrome and there is but a scant five pages dealing with other causes of acute respiratory failure. Much about the nature of the book is declared in the editors' preface in which they state that it had its origins at a meeting devoted to ARDS held in 1982, and that laboratory research on animal models forms a considerable part of the content, in spite of the dissimilarities between human and animal lungs in their response to some types of injury. The
North American flavour is apparent from the list of contributors 34 from the USA, including 15 from Boston, and from the emphasis on incidence and mortality - an estimated 50,000 deaths annually in the United States alone. The opening chapter on aetiology, demography and outcome repays careful reading because it illustrates how severity and incidence are defined and, perhaps, how practice in the UK and USA differ. Thus 1800 patients require 'prolonged mechanical ventilation' annually at the Massachusetts General Hospital (1100 beds), of whom $46 \%$ are ventilated for 1 day, and $10 \%$ suffer moderate to severe respiratory failure (ARDS), defined on the basis of radiographic findings, need for intubation or positive airway pressure, and degree of defect in oxygenation.

This introduction sets the scene for 16 chapters on aspects of structure and function in ARDS and, as might be expected, the pulmonary circulation figures prominently. Four chapters are devoted to morphology, experimental and clinical pulmonary hypertension, and radiographic investigation, with a single chapter on fluid and solute transport. Four chapters on biochemical mechanisms of injury cover the role of complement, neutrophils, oxygen radicals, neutrophil elastase, peptides and lipids, there is a rather dated chapter on blood alterations in ARDS, and an entirely laboratory based account of mechanisms for modulating collagen synthesis. More immediate practical relevance returns in the chapters by Peter Suter on respiratory mechanics in ARDS, and the analysis of oxygen exchange by Francois Lemaire and colleagues. The book concludes with two chapters devoted to cardiac and renal function and, here, too, the clinician will find a pleasing combination of theory and practical information. It is pertinent to note that a high proportion of many of the chapters is occupied by experimental findings, and that many end with an expression of uncertainty and what needs to be done in the future. This then is a book which will stimulate the inquisitive but provides little practical advice. It reflects the extent to which biochemistry and cellular physiology are taking precedence over the investigation of whole organ function and, as such, is an excellent starting point for anyone wishing to pursue these topics in the scientific literature. Quite apart from taking pleasure in its quality and mental stimulus, many will also commend the generous tribute to Myron B. Laver contributed by Henrik $\mathrm{H}$. Bendixen at the start of the volume, as well as Claude Lenfant's appreciation in his foreword of the contribution made to the entire series by Julius Comroe Jr. M. Branthwaite (London)

\title{
Announcements
}

\section{Pediatric Intensive Care Symposium}

This symposium will be held at the Sheraton Newport Hotel, Newport Beach, California, USA, from 13-14 February 1986. For further information contact: Memorial Medical Center of Long Beach-University of California, Irvine, Center for Health Education, P.O. Box 1428, Long Beach, CA 90801-1428, USA. Tel.: (213) $595-3812$

\section{4th Annual Symposium of Critical Care Medicine}

This symposium will be held at the MGM Grand Hotel, Las Vegas, Nevada, USA, from 27 February to 2 March 1986. For further information contact: Associate Dean, USC School of Medicine, Postgraduate Division, 2025 Zonal Avenue, KAM 307, Los Angeles, CA 90033, USA. Tel.: (213) 224-7051 or (800) 421-6729 (outside California) or (800) 321-1929 (within California).

4th International Symposium on Computing in Anesthesia and Intensive Care

This symposium will be held at the Doelen Concert Hall, Rotterdam, The Netherlands, from 3-6 September 1986. Main theme:
Medical instrumentation technology. Other areas: Pulmonary function in Anesthesia; Blood gas analysis; Exercise physiology; Mass spectrometry; Monitoring during Anesthesia and post-operative period; Monitoring of cardiopulmonary parameters in the intensive care units; Oxygen and carbondioxide transport; Data acquisition and display; Feedback control of ventilation; Anesthesia information management system; Cost containment program in anesthesia and intensive care; Mathematical modelling and education. Deadline for submission of abstract: 1 June 1986.

For further information write to: Omar Prakash, M.D., Erasmus University Rotterdam, P.O. Box 1738, 3000 DR Rotterdam, The Netherlands

\section{Erratum}

Intensive Care Medicine, Volume 11, Number 5, September 1985, p. 273

The name of the co-author of the letter to the editor on prostacyclin and ARDS, J. F. Searle, was inadvertently omitted. We regret this oversight. 